\title{
Results on Rare Decays and other CP Violation at the Tevatron
}

\author{
P. Squillacioti ${ }^{\text {a* }}$ \\ ${ }^{a}$ Siena University and INFN sez. Pisa, Italy.
}

We present the current status of the rare decay searches of $B_{(s)}^{0} \rightarrow \mu^{+} \mu^{-}, B \rightarrow \mu^{+} \mu^{-} h, D^{+} \rightarrow \pi^{+} \mu^{+} \mu^{-}$and $B_{(s)}^{0} \rightarrow h^{+} h^{\prime-}$ at the Tevatron Collider. We also report about the latest results on CP Violation for $B^{0}$ and $B_{s}^{0}$ decay modes into pairs of charmless charged hadrons (pions and kaons).

\section{Introduction}

Flavor changing neutral current (FCNC) decays are forbidden at tree level in the Standard Model (SM) and proceed via higher order penguin or box diagrams. Many extensions of the SM allow for tree level diagrams or alternative loop diagrams for FCNC that could significantly alter the decay rate with respect to the SM expectations. Therefore precision measurements of these rare FCNC processes are powerful probes of new physics and are complementary to direct collider searches.

At the Tevatron, we have searched for the FCNC decays of $B_{(s)}^{0} \rightarrow \mu^{+} \mu^{-}$. The SM expectation [1] for these branching fractions are significantly below the current experimental sensitivity. However, in many SUSY extensions of the SM, the branching fraction could be enhanced by one to three orders of magnitude to a level observables by the Tevatron experiments. An observation of these decay at the Tevatron would be unambiguous evidence for physics beyond the SM.

Another important FCNC process accessible at the Tevatron is the $B \rightarrow X_{s} l^{+} l^{-}$decay. New physics effects could be seen in the forwardbackward asymmetry of the strange meson in the dimuon system and/or in the decay rate [2]. At the Tevatron, we have searched for the following exclusive decay modes: $B^{+} \rightarrow K^{+} \mu^{+} \mu^{-}$, $B^{0} \rightarrow K^{(* 0)} \mu^{+} \mu^{-}$and $B_{s}^{0} \rightarrow \Phi \mu^{+} \mu^{-}$. The former two decay modes have been observed at BaBar [3] and Belle [4]. The latter decay mode

*paola.squillacioti@pi.infn.it
$B_{s}^{0} \rightarrow \Phi \mu^{+} \mu^{-}$has so far evaded experimental detection.

At the Tevatron also the FCNC charm decays are accessible. There are several scenarios of new phenomena such as SUSY R parity violation in a single coupling scheme [5], or little Higgs models with a new up-like quark [6], where deviations from the SM would only be seen in the up sector. Scenarios of this nature motivate the study of FCNC charm meson decays.

The decay modes of $\mathrm{B}$ mesons into pairs of charmless pseudo-scalar mesons are effective probes of the quark-mixing (CKM) matrix and sensitive to potential new physics effects. We report the first observation of the $B_{s}^{0} \rightarrow K^{-} \pi^{+}$ mode and a measurement of its branching fraction and direct $\mathrm{CP}$ asymmetry. We also observe for the first time two charmless decays of the $\Lambda_{b^{-}}$ baryon: $\Lambda_{b}^{0} \rightarrow p \pi^{-}$and $\Lambda_{b}^{0} \rightarrow p K^{-}$.

\section{2. $B_{(s)}^{0} \rightarrow \mu^{+} \mu^{-}$searches}

The CDF and DØ experiments use similar strategy to search for the rare decay of $B_{s}^{0} \rightarrow$ $\mu^{+} \mu^{-}$.

The offline analysis begins by selecting two muon candidates of opposite charge which satisfy the online dimuon trigger requirements. After applying some loose pre-selection requirements, the selected sample is still dominated by combinatorial background and partially reconstructed $\mathrm{B}$ hadrons. To further reduce the background a likelihood discriminant is constructed, CDF using three variables, $\mathrm{D} \varnothing$ using six variables. A likelihood ratio (LHR) is constructed as given by: 
$\frac{\prod_{i} P_{s}\left(x_{i}\right)}{\prod_{i} P_{s}\left(x_{i}\right)+\prod_{i} P_{b}\left(x_{i}\right)}$ where $x_{i}$ are the discriminant variables and $P_{s(b)}\left(x_{i}\right)$ is the probability that a signal (background) event has an observed $x_{i}$. The probability distributions for the signal events are obtained from the signal Monte Carlo (MC) and the background distributions are taken from the data sidebands.

The $B_{s}^{0} \rightarrow \mu^{+} \mu^{-}$branching fraction is obtained by normalizing to the number of $B^{+} \rightarrow$ $J / \psi K^{+} \rightarrow \mu^{+} \mu^{-} K^{+}$decays collected by the same trigger. If no signal is observed, the upper limit on the branching fraction can be computed using the expression:

$$
\begin{aligned}
& B R\left(B_{s}^{0} \rightarrow \mu^{+} \mu^{-}\right)^{90 \% C . L .}=\frac{N_{B_{s}^{0}}^{90 \%}}{N_{B^{+}}} \cdot \frac{\epsilon^{+}}{\epsilon_{B_{s}^{0}}} \cdot \frac{f_{u}}{f_{s}} . \\
& B R\left(B^{+} \rightarrow J / \psi K^{+} \rightarrow \mu^{+} \mu^{-} K^{+}\right),
\end{aligned}
$$

where $N_{B_{s}^{0}}^{90 \%}$ is the number of $B_{s}^{0} \rightarrow \mu^{+} \mu^{-}$decays at the $90 \%$ C.L. for $\mathrm{N}$ observed and $N_{B}$ expected background events. The parameter $\epsilon_{B_{s}^{0}}$ $\left(\epsilon_{B^{+}}\right)$is the total efficiency of the requirements for triggering and recostructing the signal (normalization) mode. We use the world average fragmentation ratio $f_{u} / f_{s}$ and branching fraction $B R\left(B^{+} \rightarrow J / \psi K^{+} \rightarrow \mu^{+} \mu^{-} K^{+}\right)$.

CDF optimizes the analysis based on the a priori expected $90 \%$ C.L. upper limit on $B R\left(B_{s, d}^{0} \rightarrow\right.$ $\left.\mu^{+} \mu^{-}\right)$. With the optimized selection requirements and $780 \mathrm{pb}^{-} 1$, there are 1 (2) candidates in the $B_{s}^{0} \rightarrow \mu^{+} \mu^{-}\left(B^{0} \rightarrow \mu^{+} \mu^{-}\right)$signal window, as shown in Fig. 1, with an expected background of $1.27 \pm 0.37(2.45 \pm 0.40)$. Given the number of signal events observed is consistent with the background expectation, we computed the following $90 \%(95 \%)$ C.L. of $B R\left(B_{s}^{0} \rightarrow \mu^{+} \mu^{-}\right)<8.0 \times 10^{-8}$ $\left(1.0 \times 10^{-7}\right)$ and $B R\left(B_{d}^{0} \rightarrow \mu^{+} \mu^{-}\right)<2.3 \times 10^{-8}$ $\left(3.0 \times 10^{-8}\right)$.

DØ performs two different optimization strategies using the LHR. One optimization was performed on the ratio $\epsilon_{\mu \mu} /\left\langle n_{u l}\right\rangle$, where $\left\langle n_{u l}\right\rangle$ is the expected average upper limit given the expected background in the signal region. The second method was to maximize the criterion $P=\epsilon_{\mu \mu} /\left(1+\sqrt{n_{\text {back }}}\right)[7]$. Here, $\epsilon_{\mu \mu}$ is the reconstruction efficiency of the signal MC after the pre-selection and $n_{\text {back }}$ is the expected number of background events interpolated from the sidebands. The value of the selection cuts were the same in both optimization. With the optimized selection requirements and $2 \mathrm{fb}^{-1}$, there are 1 (2) $B_{s}^{0} \rightarrow \mu^{+} \mu^{-}$candidates in Run IIa (Run IIb), as shown in Fig. 2, with an expected background of $0.8 \pm 0.2(1.5 \pm 0.3)$. The signal is compatible with the background expectation. The branching fraction limits at $90 \%(95 \%)$ C.L. is $B R\left(B_{s}^{0} \rightarrow \mu^{+} \mu^{-}\right)<7.5 \times 10^{-8}\left(9.3 \times 10^{-8}\right)$.

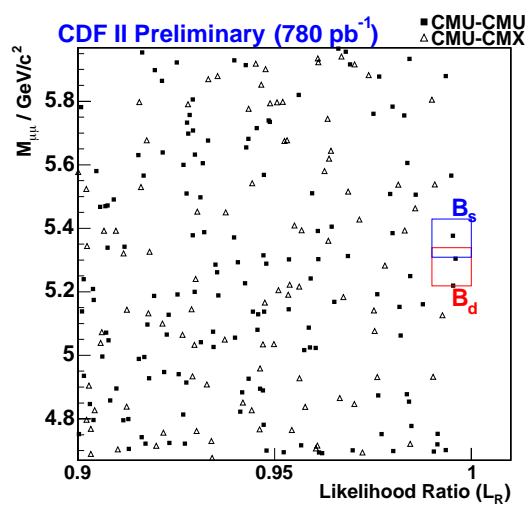

Figure 1. The $\mu^{+} \mu^{-}$invariant mass versus likelihood ratio based on $780 \mathrm{pb}^{-1}$ of CDF data, the $B_{s}^{0}$ and $B_{d}^{0}$ signal regions are also shown.

\section{Exclusive $B \rightarrow K^{(*)} \mu^{+} \mu^{-}$searches}

In addition to the two-body dimuon decays, the Tevatron has also searched for the $b \rightarrow$ $s \mu^{+} \mu^{-}$FCNC processes. In particular CDF has searched for the decays of $B^{+} \rightarrow K^{+} \mu^{+} \mu^{-}, B^{0} \rightarrow$ $K^{* 0} \mu^{+} \mu^{-}$and $B_{s}^{0} \rightarrow \Phi \mu^{+} \mu^{-}$in $1 \mathrm{fb}^{-1}$ of data. $\mathrm{D} \varnothing$ has focused the search in the $B_{s}^{0} \rightarrow \Phi \mu^{+} \mu^{-}$ channel using $450 \mathrm{pb}^{-1}$ of data. The offline analysis begins by searching for a pair of oppositely charged muon tracks. The two muon tracks are combined with a third charged track to form a $B^{+} \rightarrow K^{+} \mu^{+} \mu^{-}$candidate, or another pair of oppositely charged tracks to form a $B^{0} \rightarrow K^{* 0} \mu^{+} \mu^{-}$ 

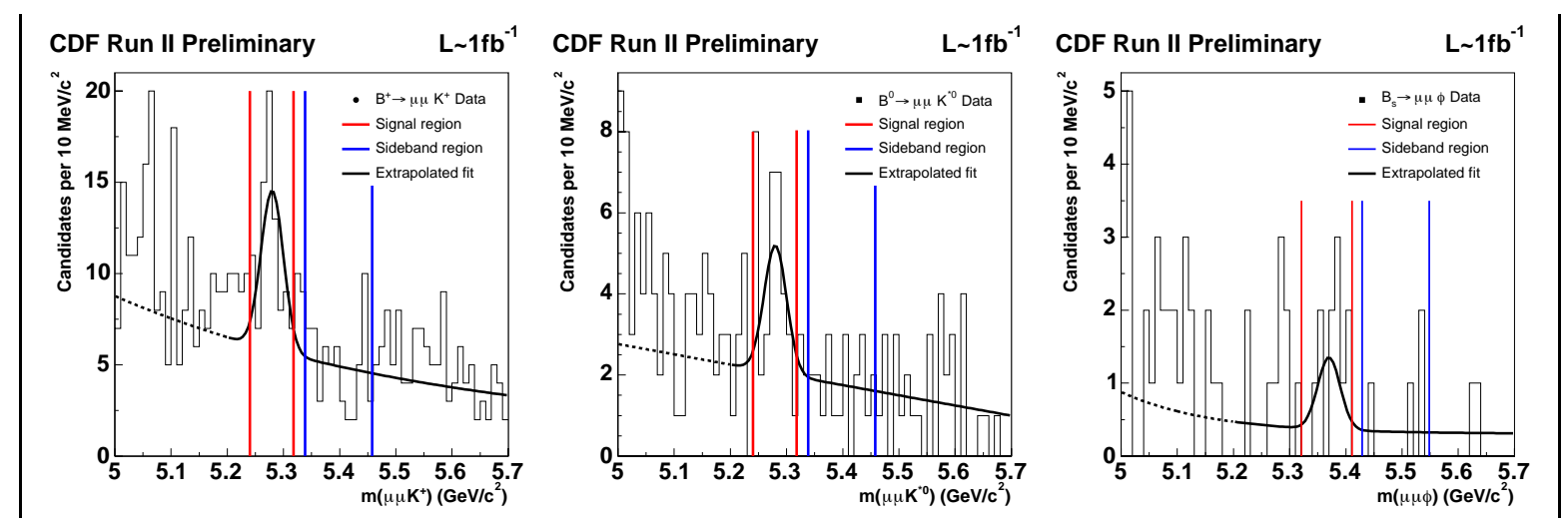

Figure 3. The invariant mass distribution for the three rare decay modes. The vertical bars define the signal and sideband regions. The black curve illustrates the expected shape for the signal and combinatoric background.

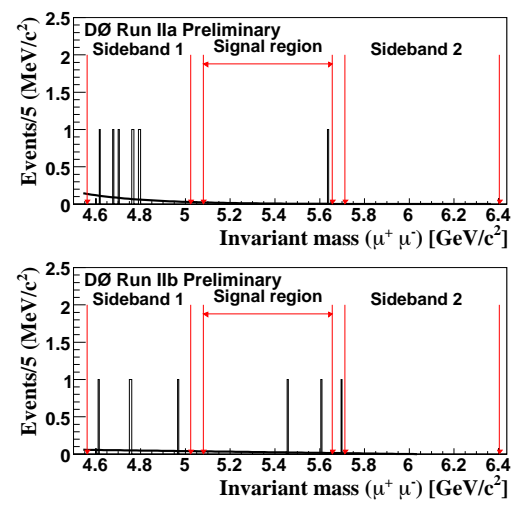

Figure 2. The dimuon mass distribution based on $2 \mathrm{fb}^{-1}$ of $\mathrm{D} \emptyset$ data.

or $B_{s}^{0} \rightarrow \Phi \mu^{+} \mu^{-}$candidate. The $K^{* 0}$ is reconstructed in the mode $K^{* 0} \rightarrow K^{+} \pi^{-}$and the $\Phi$ is reconstructed as $\Phi \rightarrow K^{+} K^{-}$. We exclude events where the dimuon invariant mass is within the $J / \psi \rightarrow \mu^{+} \mu^{-}$and $\psi(2 S) \rightarrow \mu^{+} \mu^{-}$mass regions to eliminate possible contributions from the charmonium resonant decays. CDF uses the following three discriminating variables in the optimization of the searches: the proper lifetime sig- nificance $c t / \sigma_{c t}$, the pointing angle $\alpha$ from the B meson candidate to the primary vertex and the isolation I. The expected number of background events in the B mass window is obtained by extrapolating events in the high-mass sideband to the signal region. The figure of merit for the optimization is $S / \sqrt{S+B}$ where $\mathrm{S}$ is the estimate of the expected yield of the rare decays, and $B$ is the expected background. For the $B^{+}$and $B^{0}$ rare decay searches, the PDG values of the branching fractions are used in the optimization, while the theoretical expectation is used for the $B_{s}^{0}$ search. The optimization is performed separately for the three rare decay modes. The invariant mass distribution for the three searches after applying the optimal requirements on the discriminating variables are shown in Fig. 3.

The branching fraction can be computed by normalizing the number of the observed signal to the number of reconstructed resonant $B \rightarrow h J / \psi$ decays:

$$
\begin{aligned}
& \frac{B R\left(B \rightarrow h \mu^{+} \mu^{-}\right)}{B R(B \rightarrow h J / \psi)}= \\
& \quad=\frac{N_{h \mu^{+} \mu^{-}}}{N_{h J / \psi}} \cdot \frac{\epsilon_{h J / \psi}}{\epsilon_{h \mu^{+} \mu^{-}}} \cdot B R\left(J / \psi \rightarrow \mu^{+} \mu^{-}\right),(2)
\end{aligned}
$$

where $h$ is $K^{+}, K^{* 0}$ or $\Phi$. The parameter $N_{h \mu^{+} \mu^{-}}$ is either the number of observed signal events or 


\begin{tabular}{|c|c|c|c|c|}
\hline $\begin{array}{c}\text { Experiment } \\
\text { Mode }\end{array}$ & $\begin{array}{c}\mathrm{CDF} \\
B^{+} \rightarrow \mu^{+} \mu^{-} K^{+}\end{array}$ & $\begin{array}{c}\mathrm{CDF} \\
B_{d}^{0} \rightarrow \mu^{+} \mu^{-} K^{* 0}\end{array}$ & $\begin{array}{c}\mathrm{CDF} \\
B_{s}^{0} \rightarrow \mu^{+} \mu^{-} \Phi\end{array}$ & $\begin{array}{c}\mathrm{D} \emptyset \\
B_{s}^{0} \rightarrow \mu^{+} \mu^{-} \Phi\end{array}$ \\
\hline Signal Yield & 90 & 35 & 11 & 0 \\
\hline Expected BKG & $45.3 \pm 5.8$ & $16.5 \pm 3.6$ & $3.5 \pm 1.5$ & $1.6 \pm 0.4$ \\
\hline Significance & 4.5 & 2.9 & 2.4 & - \\
\hline Rel $B R \times 10^{-3}$ & $0.59 \pm 0.15 \pm 0.03$ & $0.62 \pm 0.23 \pm 0.07$ & $1.24 \pm 0.60 \pm 0.15$ & - \\
\hline Abs $B R \times 10^{-6}$ & $0.60 \pm 0.15 \pm 0.04$ & $0.82 \pm 0.31 \pm 0.10$ & $1.16 \pm 0.56 \pm 0.42$ & - \\
\hline Rel $B R 95 \%$ CL & - & - & $2.61 \times 10^{-3}$ & $7.4 \times 10^{-3}$ \\
\hline Rel $B R 90 \%$ CL & - & - & $2.30 \times 10^{-3}$ & $5.6 \times 10^{-3}$ \\
\hline
\end{tabular}

Table 1

Summary of the $B \rightarrow h \mu^{+} \mu^{-}$searches at CDF and DØ . The branching fraction limits are computed using Bayesian method.

in the case of setting a limit, the upper limit on the number of signal decays, and $N_{h J / \psi}$ is the number of reconstructed $B \rightarrow h J / \psi$ events. The efficiency terms $\epsilon_{h J / \psi}$ and $\epsilon_{h \mu^{+} \mu^{-}}$are the efficiency for reconstructing the normalization and signal decays, respectively. The branching fraction results and limits are tabulated in Tab. 1. $\mathrm{CDF}$ has seen strong evidence of the rare decay in the $B^{+}$channel. Neither CDF nor D $\varnothing$ observed significant signal in the $B_{s}^{0}$ channel.

\section{4. $D^{+} \rightarrow \pi^{+} \mu^{+} \mu^{-}$searches}

In addition to the rare $\mathrm{B}$ decays, $\mathrm{D} \emptyset$ reported, with $1 \mathrm{fb}^{-1}$ of data, the observation of the decay $D_{s}^{+} \rightarrow \Phi \pi^{+} \rightarrow \pi^{+} \mu^{+} \mu^{-}$and the evidence for the decay $D^{+} \rightarrow \Phi \pi^{+} \rightarrow \pi^{+} \mu^{+} \mu^{-}$. The $\pi^{+} \mu^{+} \mu^{-}$invariant mass distribution after applying the optimized $D_{s}^{+}$selection is shown in Fig. 4 (top). The fit yields $65 \pm 11 D_{s}^{+}$events and $26 \pm 9 D^{+}$events with a significance above background of 3.1 standard deviations. D $\varnothing$ measured the $D^{+}$branching fraction using the central value of $D_{s}^{+} \rightarrow \Phi \pi^{+}$and $\Phi \rightarrow \mu^{+} \mu^{-}$branching fractions: $B R\left(D^{+} \rightarrow \Phi \pi^{+} \rightarrow \pi^{+} \mu^{+} \mu^{-}\right)=$ $(1.75 \pm 0.7 \pm 0.5) \times 10^{-6}$ that is in agreement with the expected value of $1.77 \times 10^{-6}$ given by the product of the $D^{+} \rightarrow \Phi \pi^{+}$and the $\Phi \rightarrow \mu^{+} \mu^{-}$ branching fractions.

DØ studied also, with the same statistics, the $c \rightarrow u \mu^{+} \mu^{-}$by looking for an excess in the $D^{+} \rightarrow \pi^{+} \mu^{+} \mu^{-}$final state in the continuum region of the dimuon invariant mass spectrum be- low and above the $\Phi$ resonance. The $\pi^{+} \mu^{+} \mu^{-}$ invariant mass distribution in data for the entire dimuon invariant mass region excluding $0.96<$ $m\left(\mu^{+} \mu^{-}\right)<1.06$ is shown in Fig. 4 (bottom). The $D^{+}$signal region contains 17 events and the combinatoric background determined from the sidebands is $20.9 \pm 3.4$. D $\varnothing$ set a limit of $B R\left(D^{+} \rightarrow \pi^{+} \mu^{+} \mu^{-}\right)<4.7 \times 10^{-6}$ at the $90 \%$ CL. This is the most stringent limit to date in these modes.

\section{Branching fractions and direct CP asymmetries of $B_{(s)}^{0} \rightarrow h^{+} h^{-}$}

CDF reconstructed a sample of about 7000 $B_{(s)}^{0} \rightarrow h^{+} h^{\prime-}\left(\right.$ where $h, h^{\prime}=K$ or $\left.\pi\right)$ in $1 \mathrm{fb}^{-1}$ of data. The $\pi \pi$-mass distributions (see Fig. 5) show a clean signal of $B_{(s)}^{0} \rightarrow h^{+} h^{\prime-}$ decays. In spite of a good mass resolution $\left(\sim 22 \mathrm{MeV} / \mathrm{c}^{2}\right)$, the various $B_{(s)}^{0} \rightarrow h^{+} h^{-}$modes overlap into an unresolved mass peak. We performed an unbinned maximum likelihood fit, combining kinematic and particle identification information to statistically determine both the contribution of each mode, and the relative contributions to the $\mathrm{CP}$ asymmetries.

We report the first observation of three new charmless decays $B_{s}^{0} \rightarrow K^{-} \pi^{+}, \Lambda_{b}^{0} \rightarrow p \pi^{+}$and $\Lambda_{b}^{0} \rightarrow p K^{+}$with a significance respectively of $8.2 \sigma, 6 \sigma$ and $11.5 \sigma$. We measured $B R\left(\Lambda_{b}^{0} \rightarrow\right.$ $\left.\left.p \pi^{-}\right) / B R\left(\Lambda_{b}^{0} \rightarrow p K^{-}\right)\right)=0.66 \pm 0.14 \pm 0.08$, in agreement with the expectations from [8]. The branching fraction of the newly observed mode 

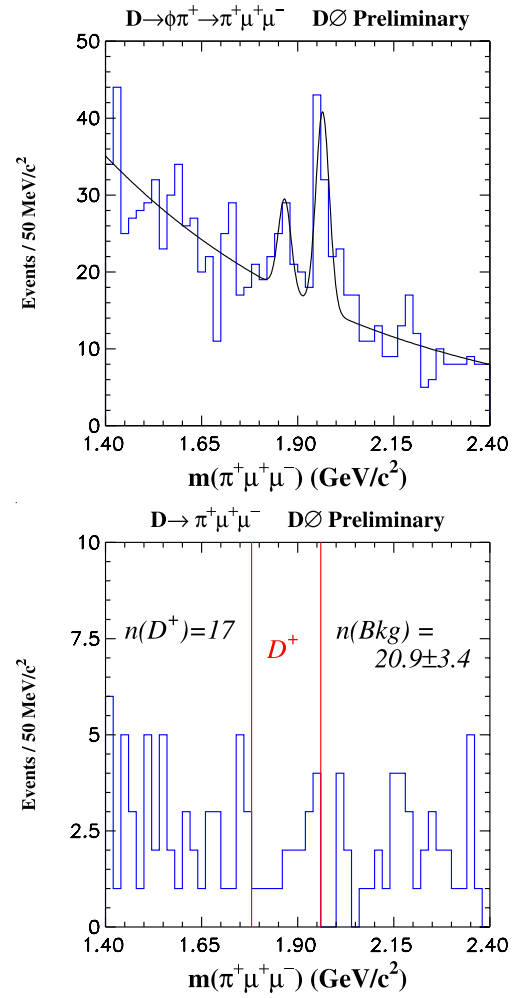

Figure 4. On the top the $m\left(\pi^{+} \mu^{+} \mu^{-}\right)$mass spectrum for the optimized $D_{s}^{+}$sample. On the bottom the final search for $D^{+} \rightarrow \pi^{+} \mu^{+} \mu^{-}$in the continuum region.

$B R\left(B_{s}^{0} \rightarrow K^{+} \pi^{+}\right)=(5.0 \pm 0.75 \pm 1.0) \times 10^{-6}$ is in agreement with the latest theoretical expectation [9]. We measured for the first time in the $B_{s}^{0}$ meson system the direct $\mathrm{CP}$ asymmetry of $A_{C P}\left(B_{s}^{0} \rightarrow K^{-} \pi^{+}\right)=0.39 \pm 0.15 \pm 0.08$. This value is in agreement with the SM expectation $\left(A_{C P}\left(B_{s}^{0} \rightarrow K^{-} \pi^{+}\right) \sim 0.37\right)$ obtained comparing the direct $\mathrm{CP}$ asymmetries in $B_{s}^{0} \rightarrow K^{-} \pi^{+}$ and $B^{0} \rightarrow K^{+} \pi^{-}$decays. The measurement $A_{C P}\left(B^{0} \rightarrow K^{+} \pi^{-}\right)=-0.086 \pm 0.023 \pm 0.009$ is the world's second best measurement and the significance of the new world average $A_{C P}^{A v g}\left(B^{0} \rightarrow\right.$ $\left.K^{+} \pi^{-}\right)=-0.095 \pm 0.013$ moved from $6 \sigma$ to $7 \sigma$.

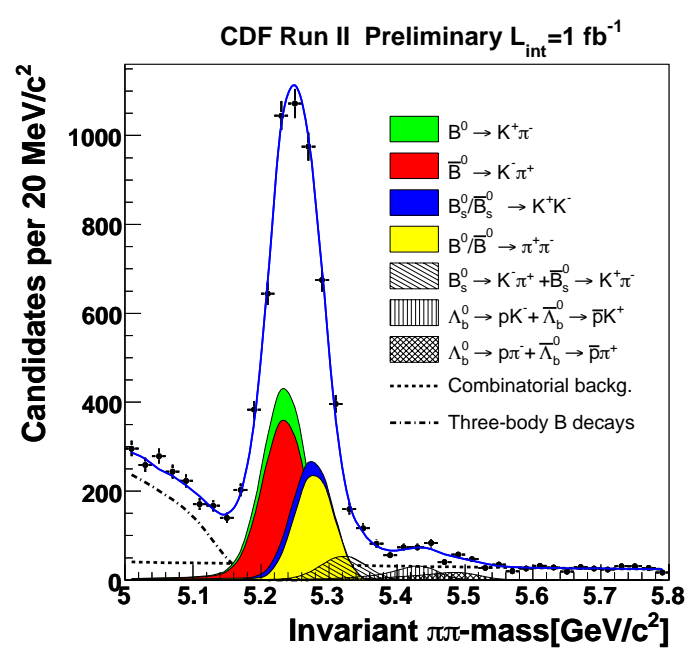

Figure 5. Invariant mass distribution of $B_{(s)}^{0} \rightarrow$ $h^{+} h^{\prime-}$ candidates using a pion mass assumption for both decay products. Comulative projections of the likelihood fit for each mode are overlaid.

\section{REFERENCES}

1. G.Buchalla and A.J.Buras, Nucl. Phys B 400, 225 (1993); A. J. Buras, Phys. Lett. B 566, 115 (2003).

2. P. Colangelo, F. De Fazio, R. Ferrandes and T. N. Pham, Phys. Rev. D 73, 115006 (2006);

F. Kruger and J. Matias, Phys. Rev. D 71, 094009 (2005); T. M. Aliev, A. Ozpineci and M. Savci, Eur. Phys. J. C 29, 265 (2003).

3. B. Aubert et al., BABAR Collaboration, Phys. Rev. D73, 092001 (2006).

4. A. Ishikawa et al., BELLE Collaboration, Phys. Rev. Lett. 96, 251801 (2006).

5. K. Agashe and M.Graesser, Phys. Rev. D54, 4445 (1996).

6. S. Fajfer and S. Perlovsek, hep-ph/0511048.

7. G. Punzi, physics/0308063.

8. R. Mohanta et al, Phys. Rev. D63, 074001 (2001).

9. A.R. Williamson, J. Zupan, Phys. Rev. D74, 014003(2006). 